\title{
Predicting observation unit treatment failures in patients with skin and soft tissue infections
}

\author{
Jon W. Schrock • Sara Laskey • Rita K. Cydulka
}

Received: 26 February 2008 / Accepted: 12 April 2008/Published online: 17 June 2008

(C) Springer-Verlag London Ltd 2008

\begin{abstract}
Background Skin and soft tissue infections are a common admission diagnosis to emergency department (ED) observation units (OU). Little is known about which patients fail OU treatment.

Aims This study evaluates clinical factors of skin or soft tissue infections associated with further inpatient treatment after OU treatment failure.

Methods A structured retrospective cohort study of consecutive adults treated for abscess or cellulitis in our OU from April 2005 to February 2006 was performed. Records were identified using ICD-9 codes and were abstracted by two trained abstractors using a structured data collection form. Significant variables on univariate analysis $P<0.1$ were entered into a multivariate logistic regression.

Results A total of 183 patient charts were reviewed. Four patients with a non-infectious diagnosis were excluded, leaving 179 patients. The median age was 41 (interquartile range: $20-74)$. Following observation treatment, $38 \%$ of patients required admission. The following variables were evaluated for association with failure to discharge home: intravenous drug use, gender, a positive communityacquired methicillin-resistant Staphylococcus aureus culture, age, presence of medical insurance, drainage of an abscess in the ED, diabetes and a white blood cell count
\end{abstract}

The views expressed in this paper are those of the author(s) and not those of the editors, editorial board or publisher.

Presented at: The American College of Emergency Physicians Scientific Assembly, New Orleans, October 2006

J. W. Schrock $(\bowtie) \cdot S$. Laskey $\cdot$ R. K. Cydulka MetroHealth Medical Center, The Department of Emergency Medicine, Case Western Reserve University School of Medicine, 2500 MetroHealth Drive, Cleveland, OH 44109-1998, USA e-mail: jschrock@metrohealth.org
(WBC) greater than 15,000. Following multivariate analysis only female gender odds ratio (OR) 2.33 [95\% confidence interval (CI): 1.06-5.15] and WBC greater than 15,000 OR 4.06 (95\% CI: $1.53-10.74)$ were significantly associated with failure to discharge.

Conclusions Among OU patients treated for skin and soft tissue infections, women were twice as likely to require hospitalization and patients with a WBC $>15,000$ on presentation to the $\mathrm{ED}$, regardless of gender, were 4 times more likely to require hospitalization.

Keywords Cellulitis · Abscess · Observation medicine · Community-acquired methicillin-resistant Staphylococcus aureus

\section{Introduction}

The epidemiology of skin and soft tissue infections seen in the emergency departments (ED) across the USA has changed considerably over the past 5 years. Skin and soft tissue infections are a common illness affecting the general population with an incidence of 24.6 per 1,000 personyears [1]. The emergence of community-acquired methicillin-resistant Staphylococcus aureus (CA-MRSA) may require a re-evaluation of treatment and disposition decisions.

Until recently, the majority of immunocompetent patients with cellulitis were treated on an outpatient basis with oral antibiotics alone. Although no definitive criteria existed to assist in determining which patients required inpatient therapy, general recommendations included admission for those who were immunocompromised, neutropaenic and exhibited signs of systemic toxicity or fasciitis [2]. For the majority of patients, clinicians must rely on their own experience and judgment when determining 
disposition. Studies published before the recent emergence of community-acquired methicillin-resistant infections noted that factors such as body part affected, diabetes and temperature were associated with the need for inpatient therapy [3-5].

A new option for treatment of these patients includes observation units [5, 6]. Observation units are often managed and staffed by emergency physicians and offer an alternative to inpatient hospital admission. These units have become more popular and are expected to become more prevalent as studies have shown significant cost savings with their use [7-10].

We attempted to clarify the role of ED observation units in the treatment of skin and soft tissue infections in the era of CA-MRSA by identifying clinical factors of skin or soft tissue infections associated with the need for further inpatient therapy after treatment in an ED and observation unit (OU).

\section{Methods}

The study was conducted at a large urban county hospital with an ED census of approximately 84,000 visits. The OU is a 14-bed unit staffed $24 \mathrm{~h}$ a day 7 days a week and managed by board certified or residency trained board prepared emergency physicians and internal medicine physicians.

Information was collected retrospectively on a cohort of consecutive adults age 18 or older admitted to our ED OU from April 2005 to February 2006 for treatment of soft tissue infection, with an international diagnostic code (ICD9 code) 680.0 through 685.1 . These codes remained valid for soft tissue infections and did not change from the 2005 to 2006 edition. Patients who were placed in the OU for a soft tissue infection but discharged with a different inflammatory condition (i.e. acute gouty arthritis) were excluded from the study. Patients who were admitted for another major condition that would require observational care, for example heart failure and cellulitis, were not included.

Records were abstracted by two trained researchers using structured review with a standardized abstraction form. The abstractors were not blinded to patient disposition when collecting data.

All data were abstracted from one of two sources. The initial source was an electronic medical record system (EPIC Systems Corporation, Madison, WI, USA) which contains all ED and outpatient clinic records, inpatient test results, operative reports, culture results, laboratory values and discharge summaries. If data were missing from the computerized chart, the original chart record was reviewed from a second computerized system (OnBase, Hyland
Software Inc., Cleveland, OH, USA) which contains the optically scanned paper records from the patient's visit. A $20 \%$ sample of medical records was reviewed by a second trained abstractor with each abstractor blinded to the other's results. Reviewer agreement was calculated using Cohen's $K$ with $95 \%$ confidence intervals $(\mathrm{CI})$.

The ED encounter during which the patients were admitted to the OU was considered the index visit. If the patient had a prior clinic or ED visit within 1 week of their index ED visit, as determined by their hospital chart, that appointment was also reviewed to determine if the skin infection was present and treated with antibiotics. All clinical encounters that occurred after the index visit were reviewed as well. Patients who were readmitted for cellulitis in the same location as the index visit within 7 days of discharge from the hospital or ED were noted as readmissions. These patients were tracked to see if poor outcomes occurred such as death and sepsis, or if length of hospitals stays were different from those patients admitted after observation treatment.

Data collected included demographics, initial vital signs, duration of symptoms, body part affected, presence of lymphangitis if mentioned, white blood cell count, absolute neutrophil count, percent bandaemia present, haemoglobin, haematocrit, initial glucose, erythrocyte sedimentation rate and C-reactive protein. Blood culture results, when performed, were recorded.

All antibiotics given prior to the index visit, at the index visit and upon discharge from the OU were recorded as well. Compliance was not assessed and therefore not addressed.

Outcomes were recorded as discharge home from the $\mathrm{OU}$, admission to the floor, admission to operating room or admission to intensive care unit. Disposition decisions were based on individual physician clinical judgment. Disposition from the OU generally occurred within $24 \mathrm{~h}$ of admission to the OU in agreement with the Department of Health and Human Services recommendations [11]. It is the policy of our OU that all patients must be out of the clinical decision unit (CDU) within $48 \mathrm{~h}$. In our institution, the number of patients in the OU for greater than $48 \mathrm{~h}$ is less than $1 \%$ of all OU patients. Any hospitalization over the next 7 days to either the hospital or OU was recorded as a readmission. Length of hospitalization was recorded in days based on number of calendar days beginning with admission from the OU to the hospital until discharge. Repeat ED visits and subsequent discharges home were not characterized as readmissions.

The timing of incision and drainage was recorded if performed. Wound culture results from the infected body part were recorded. Wound culture specimens were processed at our hospital laboratory per reported standard techniques [12]. D-zone disk diffusion tests were performed 
on all $S$. aureus isolates according to previously described techniques [13].

All data were entered into a database for analysis by the primary investigator (JS), using Stata 8.0 (StataCorp, College Station, TX, USA). Chi-squared tests and Student's $t$-test were used where appropriate.

A more moderate leukocytosis level of 15,000 was chosen a priori. We believed a more moderate leukocytosis would be more predictive of severe disease. This value has been used in other studies to assist in estimating severity of disease [14-15]. Variables thought to be clinically related to disposition decisions from the $\mathrm{OU}$ or found to be relevant in prior research were first evaluated by a fitted model for univariate analysis. Variables fitted in the univariate analysis but not used in the logistic regression due to lack of significance include African American and Hispanic race, use of a non-beta-lactam in the ED and fever. Those found to be significant were entered into a logistic regression. The variables used in the logistic regression included gender, intravenous drug use, a positive CA-MRSA culture, age, health insurance, incision and drainage procedure, diabetes and a leukocytosis greater than 15,000. Our institution's Internal Review Board reviewed the proposed study and allowed for a waiver of informed consent.

\section{Results}

A total of 183 patient charts were reviewed for inclusion into the study. Four patients who were admitted to the OU with a diagnosis of skin or soft tissue infection and were later discharged with non-infectious conditions were excluded from the reviews providing a total of 179 patients. Demographic characteristics are shown in Table 1. Forty patients $(22 \%)$ were prescribed antibiotics prior to their index visit. The median time of duration of symptoms was 3.5 days [interquartile range (IQR): 2-6 days]. Although the most common site of infection for patients requiring further inpatient treatment in the OU was the leg, patients with hand infections were most likely to require inpatient treatment (Table 2). During the study period 2,290 (1.5\%) patients were treated in the ED for skin and soft tissue infections; $179(7.8 \%)$ were admitted to the OU and 801 $(35.0 \%)$ were admitted directly to the hospital for care. A total of $64(35.8 \%)$ of the wound cultures grew CA-MRSA, which vastly outnumbered all other bacterial pathogens grown from this sample. The sensitivities for these isolates are shown in Table 3. Incision and drainage procedures were peformed in $84(47 \%)$ of the patients in the ED. Of these patients $18(11 \%)$ required a second drainage procedure after their OU treatment; $11(6 \%)$ of the patients had an initial incision and drainage procedure performed after their OU stay.
Table 1 Demographic characteristics

\begin{tabular}{lc}
\hline Characteristic & $N=179(\%)$ \\
\hline Race & $112(62.6)$ \\
Caucasian & $49(27.4)$ \\
African American & $16(8.9)$ \\
Hispanic & $2(1.1)$ \\
Asian & \\
Sex & $112(62.6)$ \\
Male & \\
Age & $82(45.8)$ \\
18-39 & $78(43.6)$ \\
$40-59$ & $19(10.6)$ \\
60 or greater & \\
Health history & $91(52)$ \\
Current smoker & $26(14.8)$ \\
IVDA & $27(15.1)$ \\
Diabetes & $4(2.2)$ \\
HIV & $98.1(97.2-99.3)$ \\
Temperature $(F)$ at ED triage & \\
WBC (cells/mm $\left.{ }^{3}\right)^{\mathrm{a}}$ & $10.73(8.3-13.5)$ \\
Abscess drained in ED & $84(47.0)$ \\
Abscess drained after OU discharge & $11(6.1)$ \\
Insurance & \\
Medicare & $22(12.3)$ \\
Medicaid & $32(17.9)$ \\
Commercial & $23(12.9)$ \\
None & $101(56.4)$ \\
Other & $2(1.1)$ \\
\hline
\end{tabular}

${ }^{a}$ Median with interquartile range

IVDA intravenous drug abuse, $H I V$ human immunodeficiency virus

In the population admitted to the OU $33(22 \%)$ had received antibiotics within 1 week prior to ED presentation. The most commonly used antibiotic class were beta-lactams used in $20(11.1 \%)$ of the patients. Sulphonamide/folate antagonists were used by $14(7.8 \%)$ of the patients. Quinolones, macrolides, tetracyclines and lincosamides were each used in $<3 \%$ of subjects prior their ED index visits. The list of antibiotics used in the ED can be seen in Table 4 . Of the patients, $18(10 \%)$ received more than one antibiotic in the ED. The antibiotics used in the ED were

Table 2 Infection site based on area of body affected

\begin{tabular}{ll}
\hline Location & Subjects, no. (\%) \\
\hline Face/head & $16(8.9)$ \\
Torso & $9(5.0)$ \\
Hand & $26(14.5)$ \\
Arm & $40(22.4)$ \\
Foot & $23(12.9)$ \\
Leg & $68(38.0)$ \\
Buttocks & $10(5.6)$ \\
\hline
\end{tabular}


Table 3 Antibiotic susceptibility (\%) among MRSA isolates

\begin{tabular}{lc}
\hline Antibiotic & MRSA $(N=64)$ \\
\hline Oxacillin & 0 \\
Erythromycin & 4.7 \\
Levofloxacin & $70.3^{\mathrm{a}}$ \\
Tetracycline & 88.9 \\
Clindamycin & $90.6^{\mathrm{b}}$ \\
TMP/SMX & 100 \\
Vancomycin & 100 \\
Rifampin & 100 \\
\hline
\end{tabular}

${ }^{a}$ Fifteen isolates showed intermediate resistance to levofloxacin

${ }^{\mathrm{b}}$ Four isolates showed inducible resistance to clindamycin

$T M P / S M X$ trimethoprim-sulphamethoxazole

also used in the OU and were prescribed by the same treating physician.

Of the patients who had documented CA-MRSA infections, 19/64 (30\%) were discharged from the OU with a prescription for a beta-lactam antibiotic; 68 (38\%) of the OU patients were admitted for further inpatient treatment. Only $4(5.3 \%)$ of the 76 patients from whom blood cultures were obtained grew bacteria: MRSA in one and methicillinsensitive Staphylococcus aureus (MSSA) in another and two contaminated culture sets. Wound cultures from each of these patients yielded the same organism. We found a high prevalence of CA-MRSA infection in this population. If contaminants are excluded, CA-MRSA constituted $78 \%$ of all positive wound cultures. One patient was admitted to the intensive care unit and one was admitted to the operating room for surgical drainage of an abscess. No patients died prior to discharge or within 7 days of discharge.

Sixty-eight patients $(38 \%)$ failed observation treatment and required inpatient admission for further therapy. Ten (5.6\%) of the patients discharged from the OU were later hospitalized within 7 days of OU discharge for further treatment of their infection. The median length of stay for patients immediately failing observation treatment was 3 days (range: 1-17) and the median length of stay of patients who were discharged after observation treatment and later admitted was 3 days (range: 1-6).

Table 4 ED antibiotics

\begin{tabular}{ll}
\hline Antibiotic classification & Subjects, no. $(\%)^{\mathrm{a}}$ \\
\hline Beta-lactam & $86(48 \%)$ \\
Vancomycin & $57(32 \%)$ \\
Lincosamide & $40(22 \%)$ \\
Sulphonamide/folate antagonist & $11(6.1 \%)$ \\
Tetracycline & $4(2.2 \%)$ \\
Quinolone & $1(0.6 \%)$ \\
\hline
\end{tabular}

${ }^{a} 10 \%$ of patients received more than one antibiotic
Length of total treatment time from ED presentation to disposition from the OU was the same for patients who were successfully treated and those who required further inpatient treatment [21.6 h (IQR: 17.9-26.0 h) vs $21.6 \mathrm{~h}$ (IQR: $16.5-25 \mathrm{~h}$ )].

Women were twice as likely to require admission [odds ratio (OR): 2.34 (1.06-5.16)] and patients with a leukocytosis greater than 15,000 were four times more likely to require admission [OR: 4.06 (1.53-10.74)] after adjusting for gender, intravenous drug use, a positive CA-MRSA culture, age, health insurance, incision and drainage procedure, diabetes and a leukocytosis greater than 15,000 . In this sample $28(16 \%)$ of the patients had a leukocytosis greater than 15,000 .

\section{Discussion}

Our data indicated that among patients admitted to an OU for skin and soft tissue infections, women and patients with a leukocytosis of greater than 15,000 were more likely to require admission after observation treatment has ended.

In this study a white blood cell count of greater than 15,000 was associated with a rate of hospital admission four times greater than baseline. This leukocytosis is likely reflective of more severe illness especially given the high proportion of CA-MRSA in our cohort. It remains uncertain how emergency physicians would use this knowledge in the management of patients with skin and soft tissue infections.

Other variables which might intuitively be associated with a higher likelihood of admission such as a positive CA-MRSA culture, diabetes or advanced age were not significantly associated with admission on multivariate analysis. This could be related to selection bias created by the limited inclusion of these patients for observation treatment or it is possible that these patients were more likely to be selected for observation treatment.

Gender disparity seen in this sample is interesting. While women were the minority of patients admitted to the OU, they were twice as likely as men to fail treatment and require hospital admission. The reason behind this is unclear. It does not appear that women were more likely to seek treatment later in the course of their illness. No other factors such as length of hospital admission seem to signify that these infections in women were more significant than in men. There does not appear to a major difference in pathogens between genders but this is only an assumption and was not an intended measure of this study. A prior study evaluating hospitalized patients with cellulitis found females to be associated with earlier discharge and a lower risk of death. The authors did not offer specific reasons for this gender difference but suggest that comorbid conditions increase the risk of complications [16]. 
The rate or mortality in this study was 5\% indicating greater severity of illness making generalization with our OU population problematic. Further research will be needed to determine if this is a local phenomenon or a trait of the management of this disease.

A surprisingly high number of blood cultures were performed in this population. As seen in other studies, the utility of this test was exceedingly low and this practice does not appear to be in line with the recommendations of the Infectious Diseases Society of America [2, 17, 18]. The admission rate for patients treated in the OU with skin and soft tissue infections of $38 \%$ is twice as high as the admission rate after treatment in our OU for other conditions such as chest pain, asthma and pneumonia. Our admission rate for cellulitis was also higher than published rates from other ED OUs [5].

The implications of the high prevalence of CA-MRSA wounds for antibiotic treatment of these infections are profound. Based on our results, patients with purulence present on exam and with a concomitant cellulitis should be provided antibiotics that cover CA-MRSA, such as trimethoprim-sulphamethoxazole, rifampin or clindamycin (which are all greater than $90 \%$ active against CA-MRSA), although clindamycin resistance of up to $60 \%$ has been found in New York City isolates [19]. For patients with a high suspicion of CA-MRSA, quinolones, macrolides and beta-lactams should be avoided as solitary coverage.

Almost one third of patients were discharged on betalactams and of those the majority did well after discharge, suggesting that incision and drainage may provide sufficient treatment for abscess. This finding is consistent with results from other investigators [19-21]. An answer to this question specifically for CA-MRSA would require prospective study to determine which if antibiotics are truly needed.

Limitations of this study include its retrospective design. We were unable to include patients who were admitted directly to the hospital from the ED and were discharged within $24 \mathrm{~h}$. This study was not intended to elucidate factors used by physicians in their decision to admit or discharge. Inherent problems may have occurred with data entry as the chart was produced. Although unlikely, incorrect DRG coding may have accidentally excluded some patients. The computerized charting system used has been suggested to improve data collection of both coded diagnosis and patient data $[22,23]$.

The population studied here contained patients considered to have moderate illness and may not be representative of all patients arriving to the ED with skin and soft tissue infections. We did not differentiate infections as nosocomial or health care-associated infections. As this was a retrospective study we could not detail if a patient was admitted to a hospital 30 days prior to their ED visit. Our OU patient population does not include nursing home patients. This population often has multiple complex medical and social issues which are not best served in our OU so these patients are typically admitted to the hospital. It would be incorrect to generalize these results for that population.

We did not perform genetic testing of our CA-MRSA isolates to determine genetic identification. Moran et al. demonstrated that risk factors for hospital-acquired infection do not correlate with genetic typing when performed. In their study, one fourth of patients possessed risk factors for hospital-acquired MRSA, yet 99\% were infected with the community-acquired strain [19].

The timing of this study occurred as the emergence of CA-MRSA was becoming more widely known. It is not known if a greater percentage of ED physicians using nonbeta-lactam antibiotics would have reduced admission.

As with all retrospective studies, we were unable to estimate how many patients discharged from the ED or OU sought further care at other institutions.

In conclusion, leukocytosis greater that 15,000 and female gender were the only variables associated with a need for further inpatient therapy after an ED observation treatment period for patients with skin and soft tissue infections. The emergence of CA-MRSA is changing how we manage skin and soft tissue infections. Increased surveillance and knowledge of the clinicians' own local bacterial resistance profiles are necessary to provide appropriate care for patients.

In conclusion we found that the majority of patients with moderate skin and soft tissue infections can successfully be treated in an ED observation setting, although the discharge rate after observation treatment is lower than other diseases such as chest pain [24-26]. Traditional risk factors for poorer outcome, including diabetes and intravenous drug abuse, in patients with skin infections did not significantly increase the odds of admission after observation treatment. In our population only leukocytosis over 15,000 and female gender significantly increased the odds of admission after observation treatment. The clinical significance of this would require further prospective studies.

Acknowledgement This study did not receive any outside funding or support.

\section{References}

1. Ellis Simonsen SM, van Orman ER, Hatch BE, Jones SS, Gren LH, Hegmann KT, Lyon JL (2006) Cellulitis incidence in a defined population. Epidemiol Infect 134:293-299

2. Stevens DL, Bisno AL, Chambers HF, Everett ED, Dellinger P, Goldstein EJ, Gorbach SL, Hirschmann JV, Kaplan EL, Montoya JG, Wade JC (2005) Practice guidelines for the diagnosis and management of skin and soft-tissue infections. Clin Infect Dis 41:1373-1406 
3. Diercks DB, Kuppermann N, Derlet RW, Ernst AA (2000) Derivation and validation of a model for the need of hospital admission in patients with extremity cellulitis. Acad Emerg Med 7:562

4. Murray HE, Stiell IG, Wells GA (2001) Defining treatment failure in ED patients with cellulitis. Acad Emerg Med 8:478

5. Raftery KA, McAfee AT, Bohan JS, Macnow LJ, Mer E (1999) Clinical predictors of the need for inpatient treatment of ED patients with cellulitis. Acad Emerg Med 6:388a

6. Roberts R (2001) Management of patients with infectious diseases in an emergency department observation unit. Emerg Med Clin North Am 19:187-207

7. Mace SE, Shah J (2002) Observation medicine in emergency medicine residency programs. Acad Emerg Med 9:169-171

8. Farkouh ME, Smars PA, Reeder GS, Zinsmeister AR, Evans RW, Meloy TD, Kopecky SL, Allen M, Allison TG, Gibbons RJ, Gabriel SE (1998) A clinical trial of a chest-pain observation unit for patients with unstable angina. Chest Pain Evaluation in the Emergency Room (CHEER) Investigators. $N$ Engl $J$ Med 339:1882-1888

9. Gaspoz JM, Lee TH, Weinstein MC, Cook EF, Goldman P, Komaroff AL, Goldman L (1994) Cost-effectiveness of a new short-stay unit to "rule out" acute myocardial infarction in low risk patients. J Am Coll Cardiol 24:1249-1259

10. Ross MA, Compton S, Kilanowski P, Medado P, O’Neil B (2006) An emergency department diagnostic protocol for patients with transient ischemic attack: a randomized controlled trial. Acad Emerg Med 13:S8a

11. Department of Health and Human Services: Center for Medicare and Medicaid Services, Medicare and Medicaid Programs; Interim and Final Rule. Federal Register, November 2007, Book 2, pp 66579-67226

12. Bannerman T (2003) Staphylococcus, Micrococcus, and other catalase-positive cocci that grow aerobically. In: Murray PR, Baron EJ (eds) Manual of clinical microbiology, 8th edn. American Society for Microbiology, Washington, DC

13. Institute CaLS (2005) Performance standards for antimicrobial susceptibility testing. 15th informational supplement, vol. 25

14. Casey JR, Marsocci SM, Murphy ML, Francis AB, Pichichero ME (2003) White blood cell count can aid judicious antibiotic prescribing in acute upper respiratory infections in children. Clin Pediatr (Phila) 42(2):113-119

15. Peltola V, Mertsola J, Ruuskanen O (2006) Comparison of total white blood cell count and serum C-reactive protein levels in confirmed bacterial and viral infections. J Pediatr 149(5):721-724

16. Carratala J, Roson B, Fernandez-Sabe N et al (2003) Factors associated with complications and mortality in adult patients hospitalized for infectious cellulitis. Eur J Clin Microbiol Infect Dis 22(3):151-157

17. Mountain D, Bailey PM, O’Brien D, Jelinek GA (2006) Blood cultures ordered in the adult emergency department are rarely useful. Eur J Emerg Med 13:76-79

18. Kelly AM (1998) Clinical impact of blood cultures taken in the emergency department. J Accid Emerg Med 15:254-256

19. Moran GJ, Krishnadasan A, Gorwitz RJ, Fosheim GE, McDougal LK, Carey RB, Talan DA (2006) Methicillin-resistant S. aureus infections among patients in the emergency department. N Engl J Med 355:666-674

20. Paydar KZ, Hansen SL, Charlebois ED, Harris HW, Young DM (2006) Inappropriate antibiotic use in soft tissue infections. Arch Surg 141:850-856

21. Lee MC, Rios AM, Aten MF, Mejias A, Cavuoti D, McCracken GH, Jr, Hardy RD (2004) Management and outcome of children with skin and soft tissue abscesses caused by community-acquired methicillin-resistant Staphylococcus aureus. Pediatr Infect Dis J 23:123-127

22. Tang PC, LaRosa MP, Gorden SM (1999) Use of computerbased records, completeness of documentation, and appropriateness of documented clinical decisions. J Am Med Inform Assoc 6:245-251

23. Stausberg J, Koch D, Ingenerf J, Betzler M (2003) Comparing paper-based with electronic patient records: lessons learned during a study on diagnosis and procedure codes. J Am Med Inform Assoc 10:470-477

24. Goodacre SW, Morris FM, Campbell S, Arnold J, Angelini K (2002) A prospective, observational study of a chest pain observation unit in a British hospital. Emerg Med J 19:117-121

25. Tatum JL, Jesse RL, Kontos MC, Nicholson CS, Schmidt KL, Roberts CS, Ornato JP (1997) Comprehensive strategy for the evaluation and triage of the chest pain patient. Ann Emerg Med 29:116-125

26. Gibler WB, Runyon JP, Levy RC, Sayre MR, Kacich R, Hattemer CR, Hamilton C, Gerlach JW, Walsh RA (1995) A rapid diagnostic and treatment center for patients with chest pain in the emergency department. Ann Emerg Med 25:1-8

Dr. Jon W. Schrock is Director of the Clinical Decision Unit and an Assistant Professor at the Case Western Reserve School of Medicine. His research interests include observation medicine, neurovascular and cardiovascular disease. 